\title{
The looming threat of bedaquiline resistance in tuberculosis
}

\author{
To the Editor:
}

Multidrug- and rifampicin-resistant tuberculosis (MDR-/RR-TB) have evolved into a global public health crisis. In late 2019, the World Health Organization (WHO) issued a rapid communication [1] advising national TB programmes to phase out the injectable-containing shorter regimen. WHO indicated that the preferred treatment option is a shorter, all-oral, bedaquiline-containing regimen and emphasised that "access to rapid drug susceptibility testing, especially for ruling out fluoroquinolone resistance, is required" before starting the bedaquiline-containing shorter regimen [1].

Phasing out injectables is simple but ensuring rapid drug susceptibility testing of fluoroquinolone is difficult in low- and middle-income countries. Rapid expansion of bedaquiline use before ruling out fluoroquinolone resistance is likely to happen in resource-limited settings where operational challenges are formidable.

The use of WHO-recommended bedaquiline-containing regimens in the treatment of MDR-/RR-TB patients with undetected resistance to fluoroquinolones runs a high risk of acquired bedaquiline resistance, especially in settings with a high prevalence of fluoroquinolone resistance [2]. A substantial proportion of MDR-/RR-TB patients who have been treated with bedaquiline has been reported to harbour bedaquiline-resistant strains in South Africa [3]. Solutions are urgently needed to avoid a looming threat of bedaquiline resistance in TB if countries decide to follow WHO's recommendation by swiftly phasing out injectables.

The first step to reduce the risk of creating an epidemic of bedaquiline-resistant TB entails addressing the lack of access to rapid fluoroquinolone drug susceptibility testing in a high proportion of MDR-/RR-TB in low- and middle-income countries. It would be safer to modify the WHO-recommended bedaquiline-containing short regimen to deal with undetected resistance of fluoroquinolone. Linezolid has been shown to be valuable in achieving sputum conversion in chronic extensively drug-resistant TB. Adding linezolid to a bedaquiline-containing short MDR-TB regimen before obtaining a fluoroquinolone test result may be a temporary option. However, adverse reactions forcing the cessation of linezolid treatment are frequent, and whether linezolid is effective in protecting bedaquiline against acquired resistance remains uncertain [4]. Furthermore, the risk of acquired resistance to linezolid, which could jeopardise its usefulness for TB and other infectious diseases, remains unknown. Since this approach has not been systematically tested before, it would be better to apply it under operational research conditions.

The alternative is to strictly follow WHO's recommendation of not using bedaquiline before obtaining fluoroquinolone susceptibility test results. It follows that the short regimen of choice is the injectable-containing short MDR-TB regimen. This has been shown to be highly effective under programme conditions [5]. It was non-inferior to the WHO recommended long regimen in the first STREAM clinical trial [6]. The use of the injectable three times per week likely might reduce the risk of ototoxicity without compromising regimen efficacy [7]. However, strict audiometric monitoring is mandatory [8], and if baseline resistance to injectable is undetected, acquired resistance to fluoroquinolone may occur.

Would it be feasible to empirically apply the WHO-recommended bedaquiline-containing regimen and subsequently modify the regimen when fluoroquinolone susceptibility test results become available? In resource-limited settings the answer is probably no. Planning to modify the regimen when such results become available is likely wishful thinking because the results will probably be unavailable for the majority of patients. Furthermore, acquired resistance to bedaquiline may have already occurred when the results

@ERSpublications

The first step to reduce the risk of creating an epidemic of bedaquiline-resistant TB entails addressing the lack of access to rapid fluoroquinolone drug susceptibility testing in a high proportion of MDR- $/$ RR-TB in low- and middle-income countries https://bit.ly/2KbtyZ3

Cite this article as: Chiang C-Y, Trébucq A, Piubello A, et al. The looming threat of bedaquiline resistance in tuberculosis. Eur Respir J 2020; 55: 2000718 [https://doi.org/10.1183/13993003.00718-2020]. 
TABLE 1 Challenges in the introduction of a bedaquiline-containing short multidrug-resistant tuberculosis (MDR-TB) treatment regimen

\begin{tabular}{|c|c|}
\hline Activities & Challenges \\
\hline $\begin{array}{l}\text { National adaptation of shorter MDR-TB regimen: } \\
\text { replacing the injectable drug with bedaquiline }\end{array}$ & $\begin{array}{l}\text { Risk of acquired resistance to bedaquiline and } \\
\text { fluoroquinolone with the adapted regimen is } \\
\text { unknown }\end{array}$ \\
\hline $\begin{array}{l}\text { DST for fluoroquinolone before starting a } \\
\text { bedaquiline-containing shorter regimen }\end{array}$ & $\begin{array}{l}\text { Obtaining DST results for fluoroquinolone in a timely } \\
\text { manner is difficult in resource-limited settings } \\
\text { Next generation sequencing that is more sensitive in } \\
\text { the detection of fluoroquinolone resistance may } \\
\text { not be available }\end{array}$ \\
\hline Ensure adherence to treatment & $\begin{array}{l}\text { Treatment interruption is common among MDR-/ } \\
\text { RR-TB patients in most settings } \\
\text { Given the long half-life of bedaquiline, the impact of } \\
\text { loss to follow-up on acquired bedaquiline } \\
\text { resistance in programme settings need to be } \\
\text { assessed }\end{array}$ \\
\hline Management of adverse drug reactions & $\begin{array}{l}\text { The use of bedaquiline, fluoroquinolone and } \\
\text { clofazimine may be associated with substantial } \\
\text { risk of QT prolongation } \\
\text { Regular assessment of QT interval could be difficult } \\
\text { in resource-limited settings }\end{array}$ \\
\hline
\end{tabular}

\#: the short bedaquiline-containing MDR-TB regimen recommended by the World Health Organization consists of levofloxacin (moxifloxacin), clofazimine, pyrazinamide and ethambutol throughout for 9-11 months, supplemented by ethionamide/prothionamide and high-dose isoniazid for the initial 4-6 months, and bedaquiline for the initial 6 months. DST: drug susceptibility testing; RR: rifampicin-resistant.

eventually become available, due to the long turnaround time for drug susceptibility testing. WHO should quickly provide guidance on the use of bedaquiline in patients without fluoroquinolone susceptibility testing. Moreover, adherence must be ensured to prevent treatment interruption which may result in bedaquiline monotherapy because of its long half-life [4].

The injectables are the main protection against acquired fluoroquinolone resistance in the injectable-containing shorter MDR-TB regimen and it is still not yet clear whether bedaquiline is as efficacious as an injectable in preventing acquired resistance to fluoroquinolones. Studies have shown that bedaquiline has a mainly bacteriostatic effect in the early treatment phase [9], and fluoroquinolone-resistant mutants may already have been selected before bedaquiline begins to kill. Furthermore, heteroresistance is relatively common with fluoroquinolones and may not be detectable without using next generation sequencing [10]. The stage two STREAM trial comparing an all-oral bedaquiline-containing regimen with an injectable-containing regimen will provide evidence on the value of bedaquiline in replacing injectables in patients with MDR-/RR-TB in whom resistance to fluoroquinolone has been excluded by line probe assay. The findings of stage two STREAM will need to be confirmed by operational studies to assess the impact of loss to follow-up on acquired bedaquiline resistance in programme settings.

WHO indicates that "phasing out injectables and replacing them with bedaquiline is an urgent priority". Evidence on bedaquiline-containing short regimens is, however scarce, with currently no data on acquired resistance to bedaquiline and fluoroquinolone. It may be wiser not to prematurely abandon injectable-containing short MDR-TB regimens before the challenges in the use of bedaquiline-containing regimen have been addressed (table 1). WHO and partners need to work closely with countries to ensure that drug susceptibility testing of fluoroquinolone and other drugs are universally available.

Chen-Yuan Chiang ${ }^{1,2,3}$, Arnaud Trébucq ${ }^{4}$, Alberto Piubello ${ }^{5}$, Hans L. Rieder ${ }^{6,7}$, Valérie Schwoebel $^{8}$ and Armand Van Deun ${ }^{3,9}$

${ }^{1}$ Division of Pulmonary Medicine, Dept of Internal Medicine, Wan Fang Hospital, Taipei Medical University, Taipei, Taiwan. ${ }^{2}$ Division of Pulmonary Medicine, Dept of Internal Medicine, School of Medicine, College of Medicine, Taipei Medical University, Taipei, Taiwan. ${ }^{3}$ International Union Against Tuberculosis and Lung Disease, Paris, France.

${ }^{4}$ Tuberculosis Consultant Services, Paris, France. ${ }^{5}$ Damien Foundation, Niamey, Niger. ${ }^{6}$ Epidemiology, Biostatistics, and 
Prevention Institute, University of Zurich, Switzerland. ${ }^{7}$ Tuberculosis Consultant Services, Kirchlindach, Switzerland.

${ }^{8}$ Toulouse, France. ${ }^{9}$ Leuven, Belgium.

Correspondence: Chen-Yuan Chiang, 111 Hsin-Long Road, Section 3, Taipei, 116, Taiwan. E-mail: cychiang@theunion.org

Received: 17 March 2020 | Accepted after revision: 10 April 2020

Author contributions: Chen-Yuan Chiang prepared the first draft of the manuscript. All authors contributed to revision of the manuscript and approved submission of the final version of the manuscript.

Conflict of interest: None declared.

\section{References}

1 World Health Organization. Rapid Communication: Key Changes to Treatment of Drug-resistant Tuberculosis. Geneva, World Health Organization, 2019. (WHO/CDS/TB/2019.26).

2 Chiang C-Y, Van Deun A, Trebucq A, et al. Multidrug-resistant tuberculosis. Lancet 2019; 394: 299.

3 Ismael N. Emerging resistance: the South African Perspective. In symposium SP-43-A3 new and repurposed drug resistance emergence: current status and prevention strategies. 1 November 2019. 50th World Conference on Lung Health of the International Union Against Tuberculosis and Lung Disease, Hyderabad, India. Int J Tuberc Lung Dis 2019; 23: Suppl. 1, S40.

4 Nimmo C, Millard J, Brien K, et al. Bedaquiline resistance in drug-resistant tuberculosis HIV co-infected patients. Eur Respir J 2020; 55: 1902383.

5 Schwœbel V, Trébucq A, Kashongwe Z, et al. Outcomes of a nine-month regimen for rifampicin-resistant tuberculosis up to 24 months after treatment completion in nine African countries. EClinicalMedicine 2020; 20: 100268.

6 Nunn AJ, Phillips PP, Meredith SK, et al. A trial of a shorter regimen for rifampicin-resistant tuberculosis. $N$ Engl J Med 2019; 380: 1201-1213.

7 World Health Organization. Technical Report on the Pharmacokinetics and Pharmacodynamics (PK/PD) of Medicines used in the Treatment of Drug-resistant Tuberculosis. Geneva, World Health Organization, 2018. (WHO/CDS/TB/2018.6).

8 Piubello A, Souleymane MB, Hassane-Harouna S, et al. Management of multidrug-resistant tuberculosis with shorter treatment regimen in Niger: Nationwide programmatic achievements. Respir Med 2020; 161: 105844.

9 Diacon AH, Dawson R, von Groote-Bidlingmaier F, et al. 14-day bactericidal activity of PA-824, bedaquiline, pyrazinamide, and moxifloxacin combinations: a randomised trial. Lancet 2012; 380: 986-993.

10 Operario DJ, Koeppel AF, Turner SD, et al. Prevalence and extent of heteroresistance by next generation sequencing of multidrug-resistant tuberculosis. PLoS One 2017; 12: e0176522. 